\title{
Traditional versus Microsphere Embolization for Hepatocellular Carcinoma: An Effectiveness Evaluation Using Data Mining
}

\author{
Pi-Yi Chang ${ }^{1}$, Chen-Yang Cheng ${ }^{2}$, Jau-Shin Hon ${ }^{3, *}$, Cheng-Ding Kuo ${ }^{3}$, Chieh-Ling Yen ${ }^{4}$ and Jyh-Wen Chai ${ }^{1}$ \\ 1 Radiology Department, Taichung Veterans General Hospital, Taichung 40705, Taiwan; \\ bibichang1023@gmail.com (P.-Y.C.); hubt@vghtc.gov.tw (J.-W.C.) \\ 2 Department of Industrial Engineering \& Management, National Taipei University of Technology, \\ Taipei 10608, Taiwan; cycheng@ntut.edu.tw \\ 3 Department of Industrial Engineering \& Enterprise Information, Tunghai University, Taichung 40704, Taiwan; \\ tintin0986325@gmail.com \\ 4 Gastrointestinal Department, Taichung Veterans General Hospital, Taichung 40705, Taiwan; \\ c9626@yahoo.com.tw \\ * Correspondence: honjs@thu.edu.tw
}

check for updates

Citation: Chang, P.-Y.; Cheng, C.-Y.; Hon, J.-S.; Kuo, C.-D.; Yen, C.-L.; Chai, J.-W. Traditional versus Microsphere Embolization for Hepatocellular Carcinoma: An Effectiveness Evaluation Using Data Mining. Healthcare 2021, 9, 929. https://doi.org/10.3390/ healthcare 9080929

Academic Editor: Pedram Sendi

Received: 10 June 2021

Accepted: 20 July 2021

Published: 23 July 2021

Publisher's Note: MDPI stays neutral with regard to jurisdictional claims in published maps and institutional affiliations.

Copyright: (c) 2021 by the authors. Licensee MDPI, Basel, Switzerland. This article is an open access article distributed under the terms and conditions of the Creative Commons Attribution (CC BY) license (https:// creativecommons.org/licenses/by/ $4.0 /)$.

\begin{abstract}
Background: For hepatocellular carcinoma ("HCC"), the current standard of treatment is hepatic artery embolization, generally through trans-catheter arterial chemoembolization ("TACE"). There are two types: traditional ("conventional" or "CTACE") and microsphere ("DC bead TACE"). Unfortunately, the literature comparing the relative effectiveness of cTACE versus DC bead TACE is inconclusive, partially due to the complexity of HCC and its response to treatment. Data mining is an excellent method to extract meaning from complex data sets. Purpose: Through the application of data mining techniques, to compare the relative effectiveness of CTACE and DC bead TACE using a large patient database and to use said comparison to establish usable guidelines for developing treatment plans for HCC patients. Materials and Methods: The data of 372 HCC patients who underwent TACE in Taichung Veterans General Hospital were analyzed. The chi-square test was used to compare the difference in the effectiveness of the two therapies was compared. Logistic regression was used to calculate the odds ratios. Furthermore, using the $\mathrm{C} 4.5$ decision tree, the two therapies were classified into applicable fields. Chi-square test, the $\mathrm{t}$-test, and logistic regression were used to verify the classification results. Results: In Barcelona Clinic Stages A and B cancers, CTACE was found to be $22.7 \%$ more effective than DC bead TACE. By using the decision tree C4.5 as a classifier, the effectiveness of either treatment for small tumors was 8.475 times than that for large tumors. DC bead TACE was 3.39 times more successful in treating patients with a single tumor than with multiple tumors. For patients with a single tumor, the chi-square test showed that 100-300 $\mu \mathrm{m}$ microspheres were significantly more effective than 300-500 $\mu \mathrm{m}$. While these findings provide a reference for the selection of an appropriate TACE approach, we noted that overall accuracy was somewhat low, possibly due to the limited population. Conclusions: We found that data mining could be applied to develop clear guidelines for physician and researcher use in the case of complex pathologies such as HCC. However, some of our results contradicted those elsewhere in the literature, possibly due to a relatively small sample size. Significantly larger data sets with appropriate levels of granularity could produce more accurate results.
\end{abstract}

Keywords: liver cancer; hepatic artery embolization; embolization prognosis; data mining; decision tree; logistic regression

\section{Introduction}

Liver and lung cancer are the two most prevalent cancers among Asian men, and Taiwan is no exception. Cancer is the leading cause of death in Taiwan, and according to its Ministry of Health and Welfare, lung and liver cancer have always remained the top two leading types of cancers, despite falling cancer rates. 
The current clinical practice for liver cancer is to treat tumors larger than $1 \mathrm{~cm}$. Before initiating treatment, the degree of cirrhosis is determined based on five clinical indicators, as it is a crucial indicator for selecting the appropriate course of treatment. The Barcelona clinic liver cancer ("BCLC") staging system is performed according to both the degree of cirrhosis (using the Child-Pugh score) and the daily physical status (using the Eastern Cooperative Oncology Group performance status scale ("ECOG PS")), along with other indicators. A simplified and summarized BCLC staging is as follows [1]:

- $\quad$ Stage 0 (very early stage)

ECOG PS 0

○ Child-Pugh A

- Stages A, B and C (early, intermediate, and advanced, respectively)

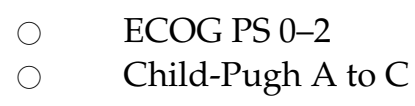

- $\quad$ Stage D (end-stage)

ECOG PS $>2$

Child-Pugh C

The hepatic artery provides $90-95 \%$ of blood and nutrition required for the survival and growth of liver cancer cells. In hepatic artery embolization, the hepatic artery is blocked to stop the blood supply to liver cancer cells; the resulting hypoxia causes shrinkage of the tumor. During embolization, chemotherapy drugs can be delivered through the catheter directly into the hepatic artery. Hepatic artery embolization is highly effective for patients with liver cancer who are not candidates for surgery, and there are lower residual levels of drugs than after conventional chemotherapy.

Currently, hepatic artery embolization is performed using two main treatment methods, both involving trans-catheter arterial chemoembolization ("TACE"). Conventional trans-catheter arterial chemoembolization ("CTACE") is the older of the two methods (Figure 1), whereas microsphere-loaded arterial embolization—called "DC bead TACE", after the drug-eluting microbeads employed-is the newer (Figure 2). DC bead TACE involves the use of a microsphere-loaded drug that prolongs effective treatment times and results in better therapeutic outcomes [2]. Compared with CTACE, DC bead TACE results in better therapeutic response and delayed tumor progression; however, no significant difference has been noted in liver-related toxicity. Song et al. demonstrated the superior performance of arterial embolization performed using DC beads [3], and Ashrafi et al. indicated that DC bead TACE can result in the same tumor response as CTACE [4]. Although the latter study combined the clinical effectiveness of DC beads with that of CTACE, additional large-scale randomized controlled trials are still needed.

According to the literature, DC bead TACE is more effective for cTACE-refractory hepatocellular carcinoma ("HCC"), particularly when the tumor is small and delayed or enhanced during angiogenesis (Figure 3). For example, Lammer et al. [5] compared the clinical efficacies of cTACE vs. DC bead TACE, both using doxorubicin, in 212 cancer patients with Child-Pugh A/B cirrhosis and large and/or multiple nodules and other inoperable cancer patients [5]. Overall, they reported that in terms of disease control, DC bead TACE was more effective than CTACE in four patients and that those adverse drug reactions were more severe after CTACE than after DC bead TACE. However, according to the chi-square analysis, the DC beads were found to have significant advantages only under certain conditions, such as a specific Child-Pugh rating and ECOG PS status, if prior curative treatments had been undergone, and in the presence of bilobar disease. 


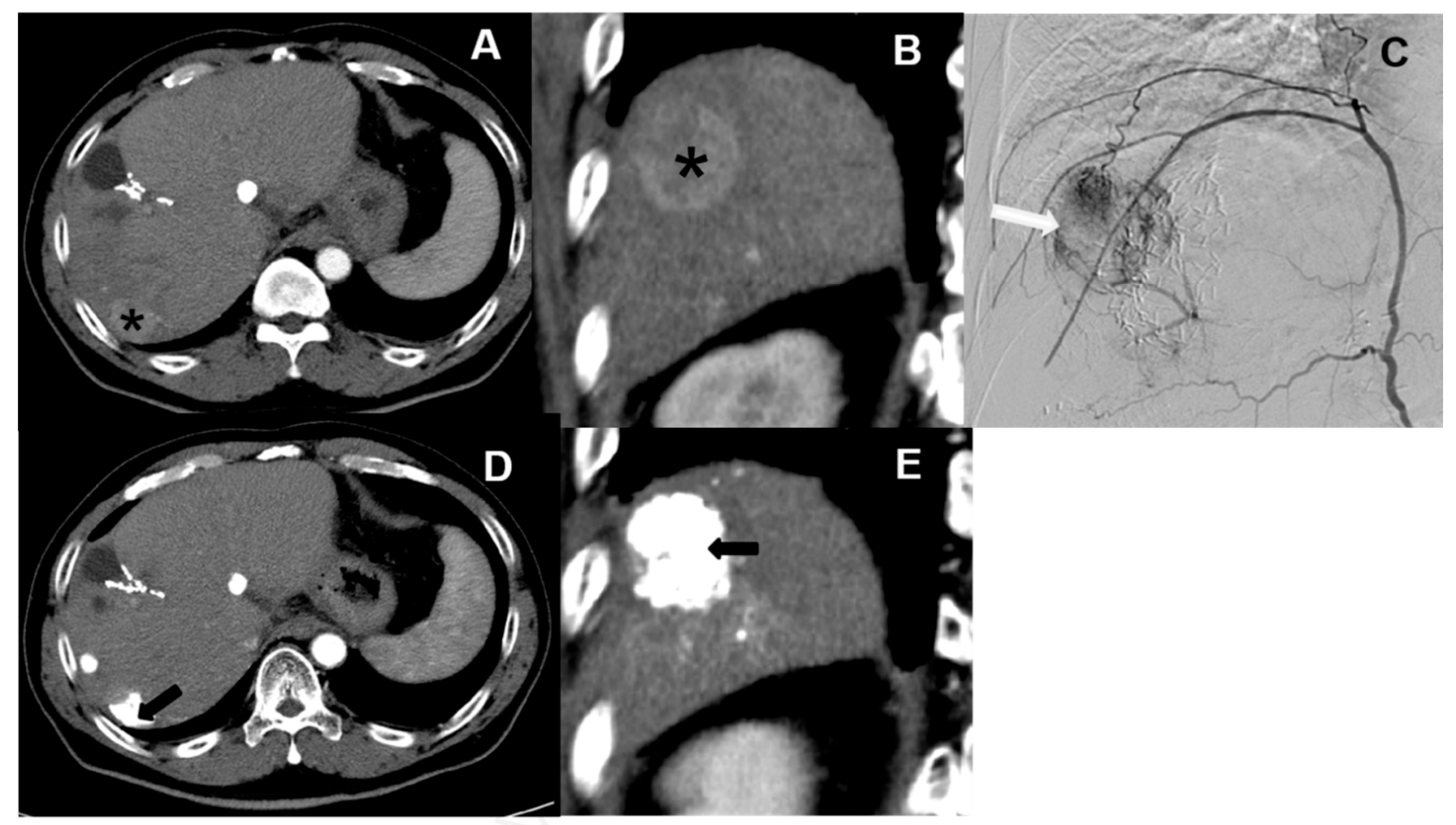

Figure 1. Traditional embolization (cTACE). Hepatocellular carcinomas (HCC) with arterial enhancement in segment 7 of the liver in both CT images (A and $\mathbf{B},{ }^{*}$ star) and angiography ( $\mathbf{C}$, white arrows). TACE is performed by using conventional TACE with lipiodol accumulation (D and E, black arrows) on follow up CT.

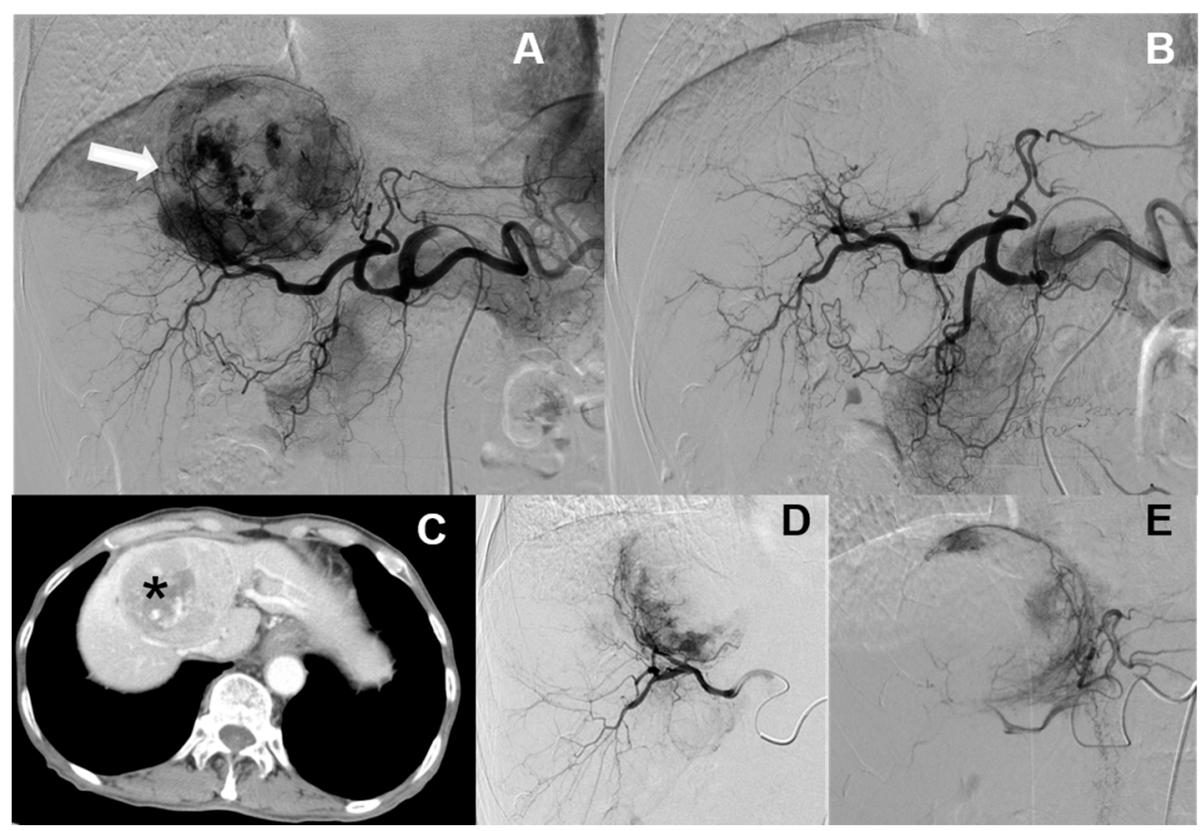

Figure 2. Microsphere embolization (DC bead TACE). A $7.3 \mathrm{~cm}$ hepatocellular carcinoma (HCC) with arterial enhancement in segment 4 and 8 of the liver in both angiography (A, white arrows) and CT $\left(\mathbf{C}^{*}{ }^{*}\right.$ star$)$ images. TACE is performed by using drug eluting microspheres (D and $\left.\mathbf{E}\right)$. Follow-up angiography shows complete embolization of the tumor (B). 


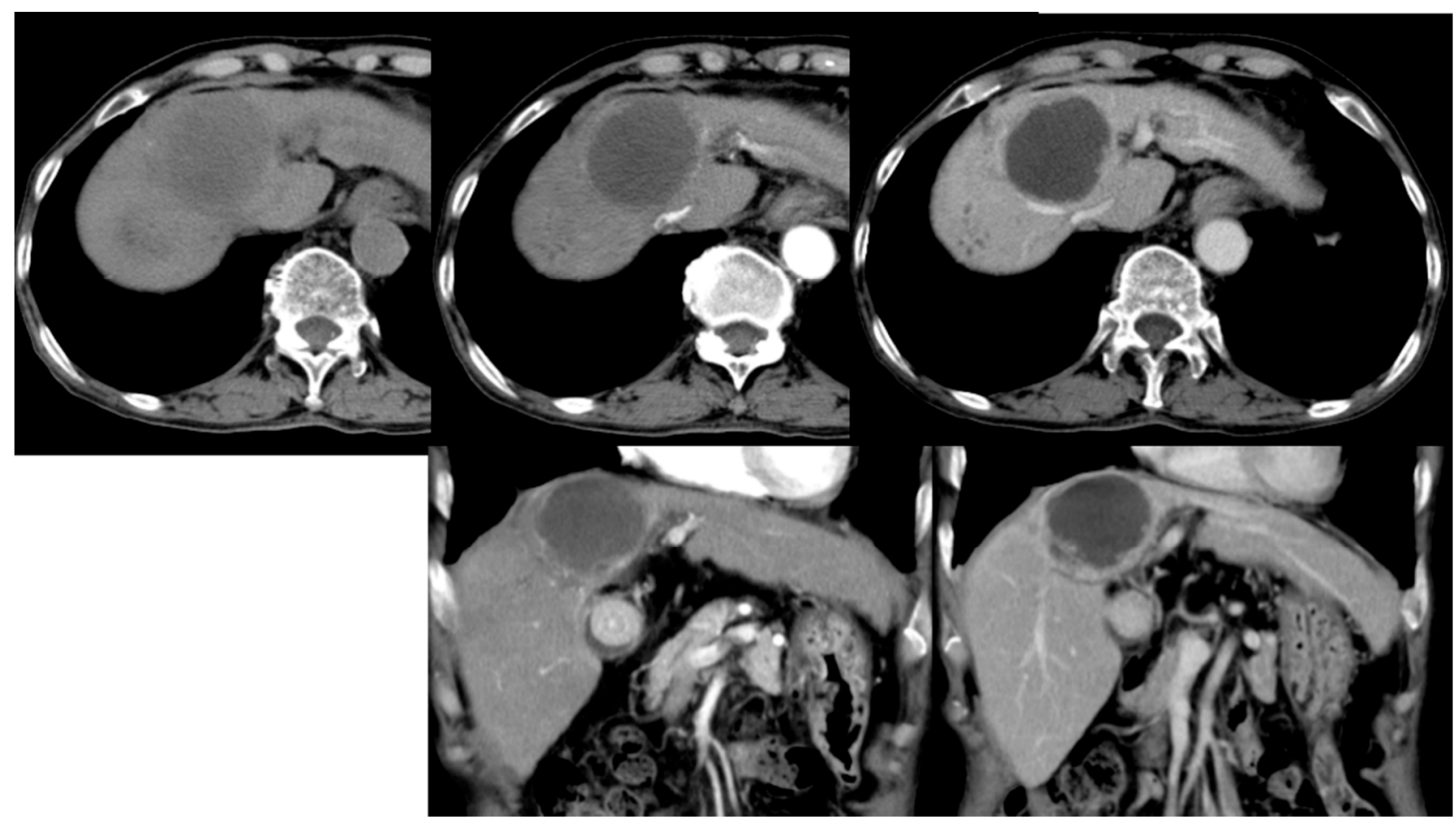

Figure 3. In a follow-up liver dynamic CT after treating with drug-eluting microspheres TACE, the tumor shows almost total necrosis.

Evaluating the clinical effectiveness of chemotherapy includes a host of other variables, complicating the analysis. For instance, Muggia et al. compared the effectiveness of cisplatinalone versus paclitaxel-alone versus combined cisplatin and paclitaxel chemotherapy in patients with late-stage ovarian cancer [6]. After patients received a 6-week treatment course, the complete and partial responses were measured every 3 weeks to determine the treatment effectiveness. Among the indicators and effects examined were neutropenia, fever, alopecia, anemia, thrombocytopenia, neurotoxicity, nephrotoxicity and gastrointestinal toxicity.

Therefore, due to the level of complexity of chemotherapy and its effects on the body, whether DC beads can completely replace cTACE in terms of curative effects remains unclear. Thus, the evaluation of which therapy is more effective under what conditions, and the identification of these conditions, can help in developing practical treatment guidelines and potential new therapies.

However, the standard practice in medical studies is to use traditional statistical analysis to determine drug effectiveness, death rates, etc. While traditional statistical analysis is adequate for finding relationships between variables and how significant those relationships are, it is not particularly well-suited for modeling complex systems in a way that is predictive in a practical sense. Rather, this is the purview of data mining and data exploration.

Data mining and exploration are a series of processes to explore the added value of information from a database by extracting and recognizing what is important or interesting in ways that cannot be known by traditional means. Data mining is also commonly known as "knowledge discovery in databases" ("KDD") and is an important tool for manipulating data to extract important information according to the user's purpose.

Data mining has become especially popular in recent years because of its ability to convert large amounts of data into some useful information and knowledge. This has been particularly useful in scientific research that employs large databases. The main difference between data mining/exploration and traditional statistics is the amount of data processed, with the former being very well-suited towards large databases. Data mining 
and exploration can create powerful predictive models of complex systems with large data sets.

The application of data mining to HCC is relatively new. A PubMed search of the terms "HCC", "liver cancer" and "data mining" yielded less than 200 results at the time of writing. The vast majority of the literature on this matter is concerned with the application of data mining to gene expression and regulation $[7,8]$, biomarkers $[9,10]$ and predictors $[11,12]$, but relatively little work has been done on the effectiveness of HCC treatments. There are a few studies regarding medications such as lenvatinib [13] and sorafenib [14], but the data-mining studies on the effectiveness of other modes of treatment are still rare.

Prior studies have shown that DC bead TACE has advantages over CTACE only under certain conditions, but due to the complexity of chemotherapy studies, clear guidelines advising the use of one over the other and under what circumstances are still lacking. This is likely due to the complexity of both HCC and its response to different modes of treatment, both of which may remain relatively opaque to traditional statistical methods. This study aims to use clinical indicators and data exploration to re-examine the effectiveness of CTACE and DC bead TACE and verify the current clinical data on liver cancer arterial embolization. This study refers to the literature on cancer prognosis [15] and employs decision trees, neural networks and logistic regressions to predict and compare data.

\section{Methods and Materials}

The present work is a retrospective study and was approved by the Institutional Review Board of Taichung Veterans General Hospital (IRB No. CE17306A), waiving the requirement for informed consent. Patient data were collected from the 2010-2017 Informatics Research and Development Center of Taichung Veterans General Hospital. We collected the data of liver cancer patients who underwent hepatic artery embolization performed using DC Bead TACE/yttrium 90 microsphere carrier drug-carrying therapy (the "new" therapy) and cTACE oil-water carrier drug therapy (the "old" therapy), as well as those of patients who underwent both the therapies. AIDS patients with severely poor prognoses were excluded from this study.

After exclusion, we collected the data of 372 patients, and defined the null hypothesis (H0) as "microsphere embolization therapy is more effective than traditional therapy" and the alternative hypothesis (H1) as "traditional therapy is more effective than microsphere embolization therapy". The Attributes or Input items of this study were liver cancer staging (unit: period), tumor size (unit: $\mathrm{cm}$ ), tumor number (unit: units) [16], new therapy/old therapy [17], microsphere size (unit: $\mu \mathrm{m}$ ) [4] and hepatitis type. The Output items were the prognostic indicators of liver cancer [15] and the effects of cancer treatment.

In the first step of logistic regression, the regression coefficient $(\beta 1,2,3 \ldots)$ was calculated from the training data; then, the probability of verification data being imported into the model with the coefficient was predicted. The predicted odds were then calculated. In medical diagnostic data mining, when the C 4.5 decision tree is compared with CART (Classification and Regression Trees), although they both exhibit a similar classification accuracy, the $\mathrm{C} 4.5$ algorithm performs better in controlling the scale of the decision tree and generates rules that are more understandable. As the object of this study was the application of medical diagnostic data mining towards evaluating the effectiveness of two different cancer therapies, the C4.5 (J48) decision tree using the ID3 system was selected.

\subsection{ID3 Algorithm}

The core concept behind the ID3 algorithm is, "the greater the information entropy, the murkier the data". The following definitions were used:

Original information entropy:

$$
I(\mathrm{p}, \mathrm{n})=-\left(\left(\frac{p}{n+p}\right) \times \log _{2}\left(\frac{p}{n+p}\right)+\left(\frac{n}{n+p}\right) \times \log _{2}\left(\frac{n}{n+p}\right)\right)
$$


Expected post processing information entropy:

$$
\mathrm{E}(\mathrm{x})=\sum_{i=1}^{n}\left(\left(\frac{n_{i}+p_{i}}{n+p}\right) x \mathrm{I}\left(n_{i}, p_{i}\right)\right)
$$

Data gain:

$$
\operatorname{Gain}(\mathrm{x})=I(\mathrm{p}, \mathrm{n})-\mathrm{E}(\mathrm{x})
$$

The data gain of each attribute is the decision parameter of the decision tree branch; that is, the maximum gain of each attribute is the branch node, indicating that the attribute can be used to minimize the turbidity of the data. The gain of all the attributes was calculated and compared.

\subsection{C4.5 (J48) Algorithm}

ID3 has a partiality problem, such as the ID number. If an ID is used for each datum as the branch point, then the gain will be maximized. Another instance is that if the selfvariation is the same in the data, then the gain will be the minimized. To prevent this issue, C4.5 changes the gain ratio to makes branch decisions. The amount of gain/self-variation of the information entropy itself (i.e., considering the problem of self-variation of the body quality) can prevent the occurrence of decision paralysis.

The core concept used herein: while adhering to the core concept of ID3, consider the information entropy and eliminate the problem of decision paralysis.

Self-variation information entropy:

$$
\operatorname{SI}(\mathrm{X})=-\sum_{j=1}^{v}\left(\left(\frac{N_{j}}{N}\right) x \log _{2}\left(\frac{N_{j}}{N}\right)\right)
$$

Information gain rate:

$$
\mathrm{GR}(\mathrm{X})=\frac{\operatorname{Gain}(\mathrm{X})}{\mathrm{SI}(\mathrm{X})}
$$

The data gain rate of each attribute is the decision parameter of the decision tree branch; that is, the maximum gain rate of each attribute is the branch node, indicating that the attribute can be divided to clarify the data as soon as possible. Finally, 10-fold cross validation is the method used to divide training and verification data. The area under the confusion matrix, the area under the receiver operating characteristic curve ("ROC curve"), and the area under the precision-recall curve ("PR curve") [18] were used and compared for the three models in this study.

From Table 1, we can understand the operation model of the overall confusion matrix and then the judgment indicators extended by the confusion matrix. We defined each indicator according to the code used in the table as follows:

Table 1. Confusion Matrix.

\begin{tabular}{l|c|c|c|c|}
\cline { 3 - 4 } \multicolumn{2}{c}{} & \multicolumn{2}{c}{ Correct } & \multicolumn{1}{c}{ Error } \\
\hline \multirow{3}{*}{ Real } & Correct & True Positive (TP) & False Negative (FN) & TP + FN = A \\
\cline { 2 - 5 } & Error & False Positive (FP) & True Negative (TN) & FP + TN = B \\
\hline & & TP + FP $=$ C & FN + TN $=\mathrm{D}$ & $\mathrm{A}+\mathrm{B}=\mathrm{C}+\mathrm{D}=\mathrm{E}$ \\
\hline
\end{tabular}

First, from the prediction accuracy surface (C, D surface):

$$
\text { Precision }=\frac{T P}{C}=\frac{T P}{T P+F P}
$$


(i.e., to predict the correct middle).

$$
\text { Recall }=\frac{T N}{D}=\frac{T N}{T P+F N}
$$

(i.e., the prediction error is in the middle).

Introduced from the real side (A, B side):

$$
\text { True positive rate }(\mathrm{TPR})=\frac{T P}{A}=\frac{T P}{T P+F N}
$$

False negative rate:

$$
(\mathrm{FNR})=\frac{\mathrm{FN}}{A}=\frac{\mathrm{FN}}{T P+F N}
$$

True negative rate:

$$
(\mathrm{TNR})=\frac{T N}{B}=\frac{T N}{F P+T N}
$$

False positive rate:

$$
(\mathrm{FPR})=\frac{F P}{B}=\frac{F P}{F P+T N}
$$

The overall model accuracy (E-side):

$$
\text { Accuracy rate }=\frac{T P+T N}{E}=\frac{T P+T N}{T P+F N+T N+F P}
$$

Precision is data taken based on a lack of information. In binary classifications, precision can be made equal to positive predictive values. Recall is deletion data that were successfully retrieved from data relevant to the query. In binary classification, recall is known as "sensitivity". The appearance of relevant data taken agrees with the query that can be seen with recall. Accuracy rate is a percentage of the total data identified and assessed. The likelihood ratio ("LR") derived from the TPR (the amount of positive data correctly classified by the system), FNR (the amount of negative data but classified incorrectly by the system), TNR (the amount of negative data correctly classified by the system), and FPR (the amount of positive data but classified incorrectly by the system) can be judged based on data calculated from the LR value.

$$
\begin{gathered}
\mathrm{LR}(+)=\mathrm{TPR} / \mathrm{FPR}=\mathrm{TP} /(\mathrm{TP}+\mathrm{FN}) / \mathrm{FP} /(\mathrm{FP}+\mathrm{TN}) \\
\mathrm{LR}(-)=\mathrm{FNR} / \mathrm{TNR}=\mathrm{FN} /(\mathrm{TP}+\mathrm{FN}) / \mathrm{TN} /(\mathrm{FP}+\mathrm{TN})
\end{gathered}
$$

The areas under the ROC and PR curves are crucial for judging the accuracy of the model. If the area is 0.5 , the probability of guessing is equal, and if the area is below 0.5 , then the probability of guessing is lower. The main difference between the two curves is the sensitivity of positive and negative samples. For example, when the positive to negative sample ratio is increased, the ROC curve does not change significantly, while the PR curve changes severely. Therefore, when differences between positive and negative samples are large, the PR curve is more suitable.

\section{Results}

\subsection{Baseline Comparison of cTACE and DC Bead TACE}

Analyses were first performed on the complete data set without any further exclusions. As shown in Table 2, the proportions of "old" and "new" treatments (cTACE and DC bead TACE, respectively) were compared with their chi-square distribution. The "cTACE is more effective" hypothesis was valid $68.2 \%$ of the time (for brevity, "effective ratio") and was invalid $31.8 \%$ of the time (for brevity, "invalid ratio"). The DC bead TACE effective ratio was $45.6 \%$ and the invalid ratio was $54.54 \%$. According to the two-tailed test, cTACE was significantly better than DC bead TACE $(p<0.05)$. 
Table 2. cTACE vs DC Bead TACE Treatments.

\begin{tabular}{|c|c|c|c|c|c|c|}
\hline & & & & & \multicolumn{2}{|c|}{ cTACE vs. DC Bead Crosstab } \\
\hline & & & & & cTACE & DC Bead \\
\hline \multirow{6}{*}{ Valid/Invalid } & \multirow[t]{3}{*}{ Invalid } & \multicolumn{3}{|c|}{ Number } & 64 & 78 \\
\hline & & \multicolumn{3}{|c|}{ Within the cTACE and DC bead treatments } & $31.80 \%$ & $54.54 \%$ \\
\hline & & \multicolumn{3}{|c|}{ Overall percentage } & $45.10 \%$ & $54.90 \%$ \\
\hline & \multirow[t]{7}{*}{ Valid } & \multicolumn{3}{|c|}{ Number } & 137 & 65 \\
\hline & & \multicolumn{3}{|c|}{ Within cTACE vs. DC bead treatments } & $68.20 \%$ & $45.60 \%$ \\
\hline & & \multicolumn{3}{|c|}{ Overall percentage } & $67.80 \%$ & $32.20 \%$ \\
\hline \multicolumn{6}{|c|}{ Chi-Square Test } & \\
\hline & & \multicolumn{2}{|r|}{ Degrees of } & Asymptotic & Precise & Precise \\
\hline & & Value & Freedom & Significant & Significant & Significant \\
\hline \multirow{2}{*}{\multicolumn{2}{|c|}{ Pearson chi-square }} & & & (two-tail) & \multirow[t]{2}{*}{ (two-tail) } & \multirow[t]{2}{*}{ (single-tail) } \\
\hline & & $17.770^{\mathrm{a}}$ & 1 & $<0.001$ & & \\
\hline \multicolumn{2}{|c|}{ Continuity correction ${ }^{b}$} & 16.845 & 1 & $<0.001$ & \multirow{5}{*}{$<0.001$} & \multirow{5}{*}{$<0.001$} \\
\hline \multicolumn{2}{|c|}{ Approximate ratio } & 17.793 & 1 & $<0.001$ & & \\
\hline \multirow{2}{*}{\multicolumn{2}{|c|}{$\begin{array}{l}\text { Fisher's accurate veritication } \\
\text { Linear connection }\end{array}$}} & & \multirow[b]{2}{*}{1} & \multirow{3}{*}{$<0.001$} & & \\
\hline & & 17.718 & & & & \\
\hline The va & observation number & 344 & & & & \\
\hline
\end{tabular}

a The expected number of 0 grid $(0 \%)$ was less than 5 , and the minimum expected number was 59.03 . $^{\mathrm{b}}$ could only calculate $2 \times 2$ forms.

To evaluate the impact of BCLC cancer staging on effectiveness, the data of patients with either BCLC stage C or D cancer were excluded; then, the effectiveness of the two treatments was compared. The number of patients who underwent CTACE did not change, indicating that patients with either stage C or D cancer did not undergo the "old" embolization therapy. Thus, the cTACE effective ratio did not change. However, the number of patients who underwent the new therapy changed from 171 to 143 , a difference of 28 patients with stage $\mathrm{C}$ or D cancer. However, the DC bead TACE effective ratio only changed from $45.6 \%$ to $45.5 \%$. Thus, no significant differences were observed in the effectiveness of old and new therapies for patients with stage $C$ or D cancer. The effective ratios of patient pools with stage $\mathrm{C}$ or $\mathrm{D}$ cancer were similar to those without, indicating a significant advantage of cTACE for patients with stage A or B cancer. The results of the logistic regression are shown in Table 3, which shows the regression coefficients for each group.

Table 3. Logistic regression output results (odds ratios expressed as regression coefficients).

\begin{tabular}{cc}
\hline Variable & Class Valid \\
\hline Hepatitis $=$ B & 0.8758 \\
Hepatitis = C & 1.1042 \\
Hepatitis = non-B non-C & 0.9071 \\
Hepatitis = B + C & 1.3726 \\
BCLC stage = B & 0.9389 \\
BCLC stage $=$ C & 1.2431 \\
BCLC stage = A & 0.9672 \\
BCLC stage $=$ D & 1.7284 \\
Quantity $=$ single & 2.8719 \\
Size & 0.9137 \\
Microsphere $=300-500$ & 0.2855 \\
Microsphere $=300-500+500-700$ & 1.5049 \\
Microsphere $=100-300$ & 0.5364 \\
Microsphere $=500-700$ & 0.4341 \\
Microsphere $=100-300+500-700$ & $9.99 \times 10^{19}$ \\
Microsphere $=100-300+300-500$ & $46,749,492.226$ \\
Microsphere $=0$ & 1.0669 \\
New vs. old treatments & 1.0669 \\
\hline
\end{tabular}




\section{2. cTACE Limitations and Applicable Segments}

The C4.5 decision tree and real medical records were used to determine whether tumor size affected the effectiveness of either treatment on HCC. Tumors were considered "small" if they were $9.3 \mathrm{~cm}$ or smaller and were "large" otherwise. We began by examining the effect of tumor size using the statistical mean $t$ test. The average tumor size that showed an effective curative effect was compared with that which did not show one, and chi-square test results are shown in Table 4 . Therefore, the results implied that if cancer is graded using the BCLC staging system, those with stages A and B cancer can undergo cTACE if the tumor size is $9.3 \mathrm{~cm}$ or smaller and that other treatments should be recommended if the tumor size is larger than $9.3 \mathrm{~cm}$.

Table 4. T-test result, Tumor Size vs. Effectiveness.

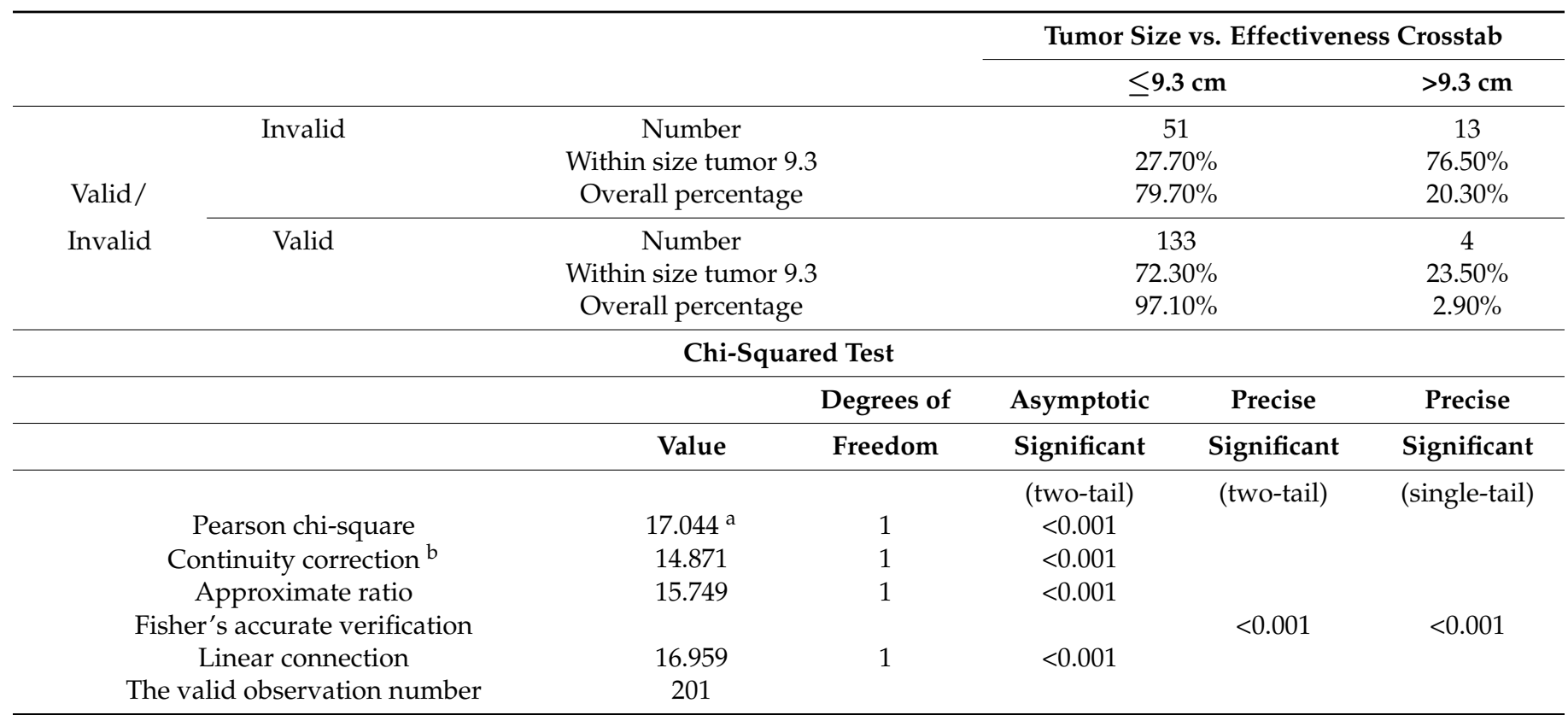

a The expected number of 0 grid $(0 \%)$ is less than 5 and the minimum expected number is $5.41 .{ }^{\mathrm{b}}$ could only calculate $2 \times 2$ forms.

Logistic regression was used to compare the difference in effectiveness between the two treatments. The odds ratios of the logistic regression output indicated that the rate of effectiveness for small tumors was 8.475 times better than the large tumors. Therefore, if the tumor size is $9.3 \mathrm{~cm}$ or smaller in patients with stage A or B cancer, then they should undergo cTACE. If the tumor is larger than $9.3 \mathrm{~cm}$, then the success rate of CTACE drops to $11.8 \%$. This result could provide a reference or guideline for oncologists to choose the most appropriate therapy.

The effectiveness of DC bead TACE (aka "microsphere therapy") was determined using a C4.5 decision tree. The model was divided into two layers. The first node was divided by the number of tumors, and the second node was divided by the size of the ball. A preliminary analysis showed that the decision tree could find that different sizes of microspheres affected the effectiveness of treatment. However, the accuracy of the model was $59.1 \%$, the false positive rate was 0.41 , the accuracy was 0.594 , and the recall rate was 0.591 . The prediction ability of the area under the ROC curve and the area under the PR curve surface were 0.6 and 0.438 , respectively.

Because the accuracy of the first iteration was so low, the mixed-sized microsphere cases were then excluded from the next iteration. This was done because there were very few cases using mixed-size microspheres. The accuracy of the model improved from $59.1 \%$ to $65 \%$. Compared with the model tree in mixed-sized microspheres, the scale of the model tree was substantially decreased. The applicable conditions of the model are shown in Figure 4 . 


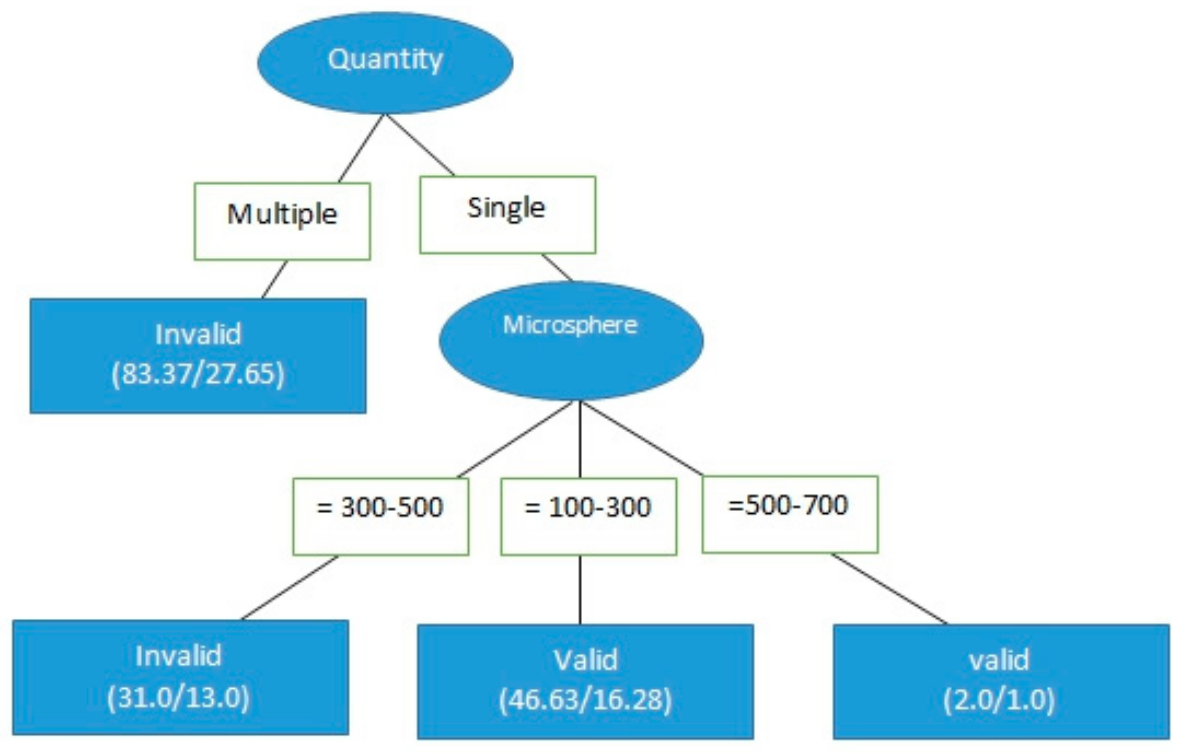

Figure 4. Decision tree classification of the effectiveness of DC bead TACE with single-sized microspheres on single tumors.

To further optimize the model, the data were examined for further possible small but potentially significant exclusions. Microspheres of 500-700 $\mu \mathrm{m}$ in size were used only in 5 patients out of the original 130 who received DC bead TACE, and so they were excluded as well. The classification results are shown in Figure 5. The accuracy of the model increased from $65 \%$ to $70.4 \%$, and those of other overall indicators also increased sharply. These findings indicated that the amount of attribute data alone did not affect the accuracy of the model.

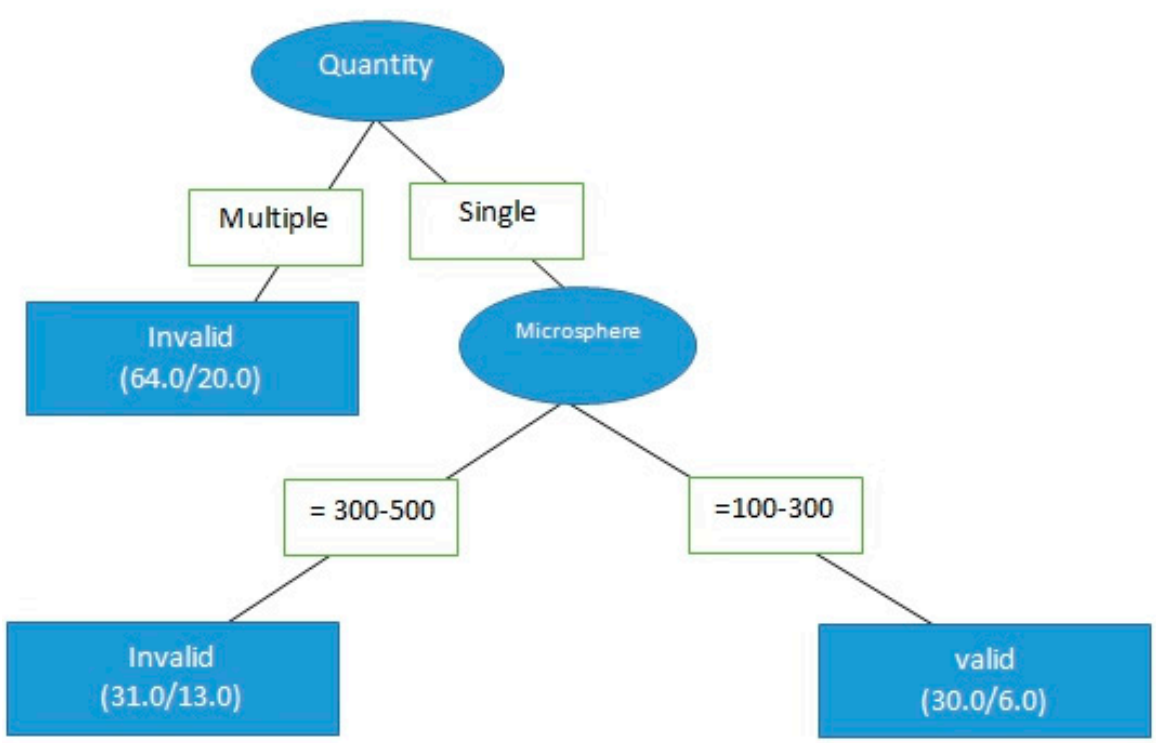

Figure 5. Decision tree classification, the same as the previous iteration but with $500-700 \mu \mathrm{m}$-sized microspheres also excluded.

With these exclusions in place, the chi-square test was used to examine the differences in the effectiveness of tumor treatment, with the degree of difference determined using logistic regression. Then the chi-square test and logistic regression were performed for microsphere sizing according to the results of the decision tree classification, in order to provide a more complete result for medical reference. The results of the chi-square test for DC bead TACE treatment are shown in Table 5 . The single-tumor treatment invalid ratio 
was $39.3 \%$, whereas the effective ratio was $60.7 \%$. The invalid ratio of multiple tumors was $68.8 \%$, whereas the effective ratio was $31.3 \%$, and there was a significant difference between the single-tumor and multiple-tumor (chi-square test $=0.01$ ).

Table 5. Microsphere treatment (DC bead TACE), excluding mixed-sized and 500-700 $\mu \mathrm{m}$-sized microspheres.

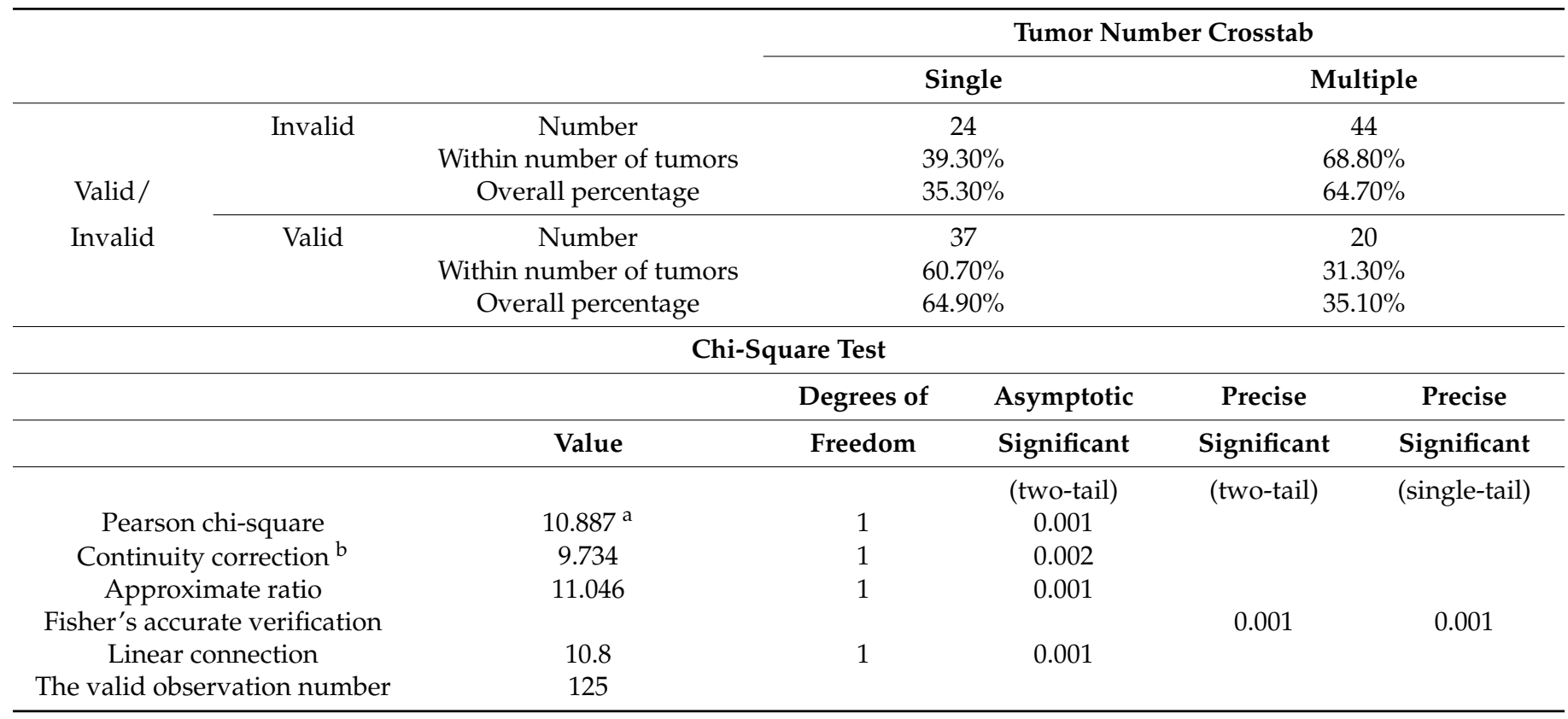

a The expected number of 0 grid $(0 \%)$ was less than 5 and the minimum expected number is $27.82 .{ }^{\mathrm{b}}$ can only calculate $2 \times 2$ forms.

\section{Discussion}

\subsection{Baseline Comparison of cTACE and DC Bead TACE}

In terms of cTACE and DC bead TACE comparison, this result differed from those of previous studies. In the present study, the difference between the validity of the two hypotheses was $22.7 \%$ in favor of the "cTACE is more effective" hypothesis. This statistical result shows that the old CTACE method has a therapeutic advantage, but with the caveat of it only occurring when the stage of cancer is not considered.

To evaluate the impact of BCLC cancer staging on two treatments, the regression coefficients of the traditional therapy showed that it is 1.0669 times more effective than that of the microsphere therapy. The regression coefficients of DC bead TACE mixed-sized microsphere groups of 100-300 $\mu \mathrm{m}+300-500 \mu \mathrm{m}$ and 100-300 $\mu \mathrm{m}+500-700 \mu \mathrm{m}$ in size were relatively high. Data on patients with multiple tumors and their sizes and locations were not available. In both cases, there is a high probability of producing effective results with more adequate clinical data. In the future, it may be possible to show that the number of tumors (as opposed to single vs. multiple) is a more influential factor in the effectiveness of both treatments and to explore the effectiveness of mixed-sized microspheres in DC bead TACE. These results indicate two possibilities. First, patients with stage A or B cancer who undergo cTACE have a high probability of controlling their cancer, and patients with stage $C$ or D cancer do not undergo cTACE. Therefore, we could not compare the advantages and disadvantages of the old and new treatments in patients with stage $C$ or D cancer and suggest this be an area of future research. The second possibility is that although microsphere therapy provides the benefit of expanding the field of liver cancer embolization, its treatment effect could be relatively low. The results above show an effectiveness rate of only $45.6 \%$ overall and administering this therapy to cancer patients with congenital conditions is difficult. Even after excluding cancer patients with congenital conditions who are difficult to treat, and patients with stage $C$ or $D$ disease, microsphere therapy still showed a low relatively low therapeutic effect, which could also be a topic for further study. 


\section{2. cTACE Limitations and Applicable Segments}

The C4.5 decision tree and real medical records were used to determine whether tumor size affected the effectiveness of either treatment on HCC. These results indicated that microsphere therapy has significant advantages in the treatment of single-tumor patients. More research into the risks and effectiveness of DC bead TACE in multiple-tumor patients is necessary, particularly in terms of the question of whether the number of tumors impacts effectiveness.

Logistic regression was then used to calculate the odds ratio of the number of tumors affecting treatment effectiveness. Treating a single tumor was 3.39 times more successful than treating multiple tumors. However, we recognized that this odds ratio was calculated from a fairly limited data set. Although the accuracy of the model was approximately $70.4 \%$, the prediction ability was not very satisfactory. Having more clinical data in the future is necessary to improve model prediction.

Next, only the data of the 61 patients who were treated for single-tumor HCC with DC bead TACE using either 100-300 $\mu \mathrm{m}$ or $300-500 \mu \mathrm{m}$ microspheres were analyzed to determine the effect of microsphere size on treatment effectiveness. The data were divided into two different microsphere sizes (30 records for 100-300 $\mu \mathrm{m}$ and 31 records for $300-500 \mu \mathrm{m}$, as per Figure 2), for a total of 61 records. The result of the chi-square test is shown in Table 6. For microspheres that were $100-300 \mu \mathrm{m}$ in size, the invalid ratio was $20 \%$ and the effective ratio was $80 \%$. For microspheres that were $300-500 \mu \mathrm{m}$ in size, the invalid ratio was $58.1 \%$, whereas the effective ratio was $41.9 \%$; the chi-square test result was 0.02 , indicating a significant difference, that is, microspheres sized $100-300 \mu \mathrm{m}$ were more therapeutically effective than those sized $300-500 \mu \mathrm{m}$ in size for a single tumor. The extent of the differences in effectiveness should be further explored.

Table 6. Microsphere therapy, single-tumor only, mixed-size and 500-700 $\mu \mathrm{m}$-sized microspheres excluded.

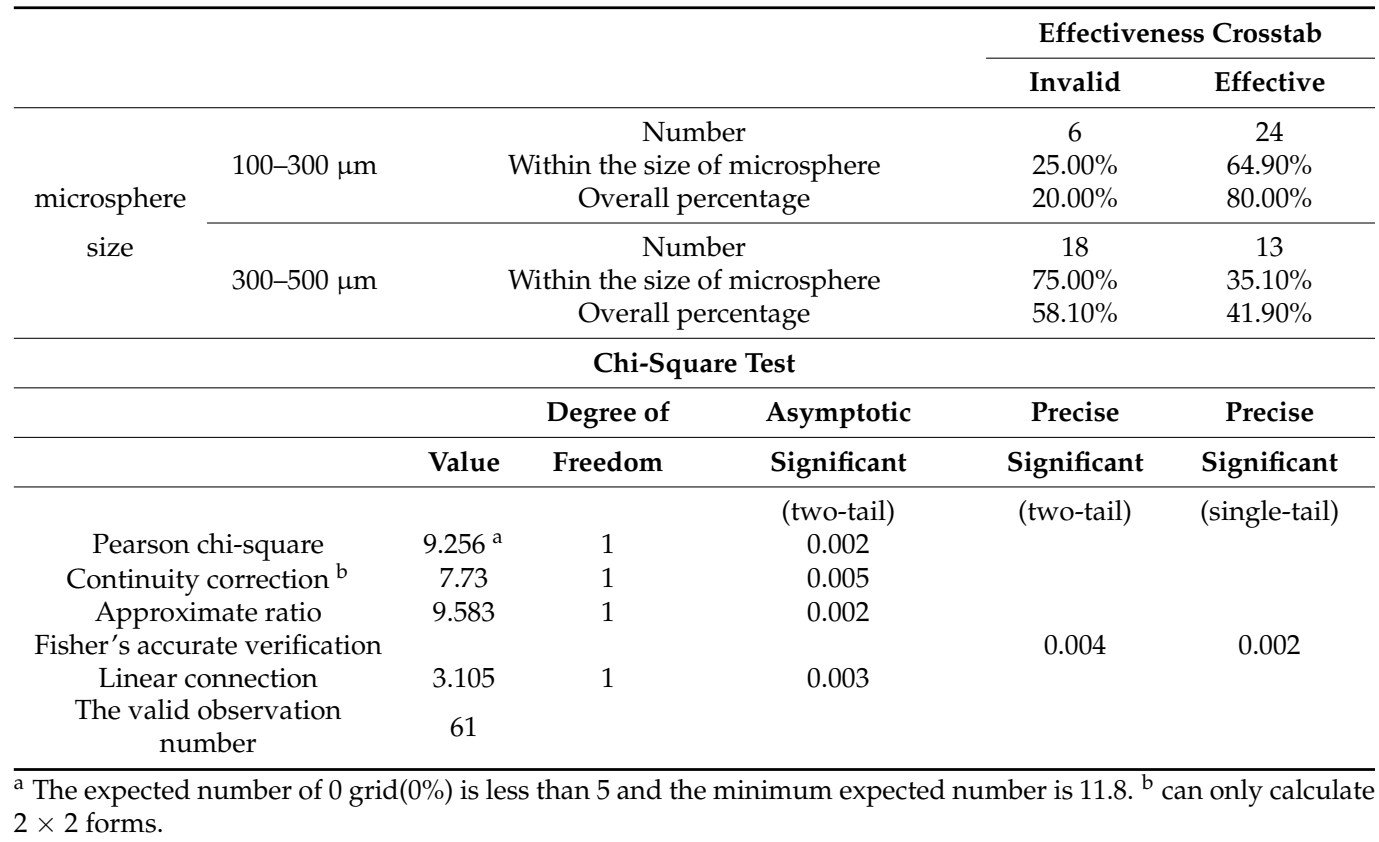

The odds ratios of the microspheres that were 100-300 and 300-500 $\mu \mathrm{m}$ in size were calculated using a logistic regression. The success rate of treatment with DC bead TACE using 100-300 $\mu \mathrm{m}$ microspheres was 3.412 times of that using 300-500 $\mu \mathrm{m}$ microspheres. The accuracy of this model was approximately $64.8 \%$; however, its prediction ability still needs to be strengthened.

One limitation of this study was the relatively small sample size. Although the factors surrounding HCC and TACE are varied and complex, the strengths of data mining lie also in its ability to analyze large data sets. While the initial population was reasonably large (372), 
by the time the analysis had reached the point of parsing different aspects of microsphere therapy, the populations had shrunk considerably. In the future, larger populations to which data mining can be applied would probably yield more interesting results.

\section{Conclusions}

This study found that the effective rate of the traditional CTACE therapy is 1.0669 times better than that of the DC bead TACE microsphere therapy. The effectiveness of either treatment is also affected by tumor size; tumors $9.3 \mathrm{~cm}$ or smaller responded much better treatment than those that were larger. Furthermore, microsphere therapy was found to be 2.8719 times more effective in treating single tumors than multiple tumors. Microspheres that were $100-300$ and $300-500 \mu \mathrm{m}$ in size had a high effective probability, and their effectiveness should be verified in future studies. Therefore, for patients with stage A or B HCC, the use of CTACE is supported by our data, showing a significant advantage of $22.7 \%$. The data also support the treatment paradigm that patients with stage $\mathrm{C}$ or $\mathrm{D}$ HCC respond better to DC bead TACE, with the caveat that having multiple tumors has a negative impact on effectiveness. The data also indicate that microspheres larger than $500 \mu \mathrm{m}$ are not as effective as smaller microspheres. As a result, we conclude that data mining techniques can be effectively used to evaluate treatment effectiveness and create clear clinical guidelines for physicians. However, larger data sets are recommended for better accuracy.

Author Contributions: Conceptualization and methodology, P.-Y.C. and C.-Y.C.; data curation, P.-Y.C. and C.-L.Y.; writing — original draft preparation, P.-Y.C. and C.-D.K.; writing—review and editing, C.-Y.C.; supervision, J.-S.H. and J.-W.C.; project administration, J.-S.H. All authors have read and agreed to the published version of the manuscript.

Funding: This research received no external funding.

Institutional Review Board Statement: This retrospective study was approved by the Institutional Review Board of Taichung Veterans General Hospital (IRB No. CE17306A), waiving the requirement for informed consent.

Informed Consent Statement: The IRB of Taichung Veterans General Hospital waived the requirement for informed consent because this was a retrospective study.

Conflicts of Interest: J.-S.H. declares that he has no conflict of interest. P.-Y.C. declares that she has no conflict of interest. C.-Y.C. declares that he has no conflict of interest. C.-D.K. declares that he has no conflict of interest. C.-L.Y. declares that she has no conflict of interest. J.-W.C. declares that he has no conflict of interest.

Ethical Approval: This article does not contain any studies with human participants or animals performed by any of the authors.

\section{References}

1. Planas, R.; Ballesté, B.; Alvarez-Gonzalez, M.A.; Rivera, M.; Montoliu, S.; Galeras, J.A.; Santos, J.; Coll, S.; Morillas, R.M.; Solà, R. Natural history of decompensated hepatitis C virus-related cirrhosis. A study of 200 patients. J. Hepatol. 2004, 40, 823-830. [CrossRef] [PubMed]

2. Nam, H.C.; Jang, B.; Song, M.J. Transarterial chemoembolization with drug-eluting beads in hepatocellular carcinoma. World J. Gastroenterol. 2016, 22, 8853-8861. [CrossRef] [PubMed]

3. Song, D.S.; Choi, J.Y.; Yoo, S.H.; Kim, H.Y.; Song, M.J.; Bae, S.H.; Yoon, S.K.; Chun, H.J.; Gil Choi, B.; Lee, H.G. DC Bead Transarterial Chemoembolization Is Effective in Hepatocellular Carcinoma Refractory to Conventional Transarteral Chemoembolization: A Pilot Study. Gut Liver 2013, 7, 89-95. [CrossRef] [PubMed]

4. Ashrafi, K.; Tang, Y.; Britton, H.; Domenge, O.; Blino, D.; Bushby, A.J.; Shuturminska, K.; Hartog, M.D.; Radaelli, A.; Negussie, A.H.; et al. Characterization of a novel intrinsically radiopaque Drug-eluting Bead for image-guided therapy: DC Bead LUMI ${ }^{\mathrm{TM}}$. J. Control Release 2017, 250, 36-47. [CrossRef] [PubMed]

5. Lammer, J.; Malagari, K.; Vogl, T.; Pilleul, F.; Denys, A.; Watkinson, A.; Pitton, M.; Sergent, G.; Pfammatter, T.; Terraz, S.; et al. Prospective Randomized Study of Doxorubicin-Eluting-Bead Embolization in the Treatment of Hepatocellular Carcinoma: Results of the PRECISION V Study. Cardiovasc. Interv. Radiol. 2009, 33, 41-52. [CrossRef] [PubMed] 
6. Muggia, F.; Braly, P.S.; Brady, M.F.; Sutton, G.; Niemann, T.H.; Lentz, S.L.; Alvarez, R.D.; Kucera, P.R.; Small, J.M. Phase III Randomized Study of Cisplatin versus Paclitaxel Versus Cisplatin and Paclitaxel in Patients with Suboptimal Stage III or IV Ovarian Cancer: A Gynecologic Oncology Group Study. J. Clin. Oncol. 2000, 18, 106. [CrossRef] [PubMed]

7. Lin, Y.; Liang, R.; Qiu, Y.; Lv, Y.; Zhang, J.; Qin, G.; Yuan, C.; Liu, Z.; Li, Y.; Zou, D.; et al. Expression and gene regulation network of RBM8A in hepatocellular carcinoma based on data mining. Aging 2019, 11, 423-447. [CrossRef] [PubMed]

8. Zou, H.; Liao, M.; Xu, W.; Yao, R.; Liao, W. Data mining of the expression and regulatory role of BCAT1 in hepatocellular carcinoma. Oncol. Lett. 2019, 18, 5879-5888. [CrossRef] [PubMed]

9. Zhang, R.; Ye, J.; Huang, H.; Du, X. Mining featured biomarkers associated with vascular invasion in HCC by bioinformatics analysis with TCGA RNA sequencing data. Biomed. Pharmacother. 2019, 118, 109274. [CrossRef] [PubMed]

10. He, G.; Fu, S.; Li, Y.; Li, T.; Mei, P.; Feng, L.; Cai, L.; Cheng, Y.; Zhou, C.; Tang, Y.; et al. TCGA and ESTIMATE data mining to identify potential prognostic biomarkers in HCC patients. Aging 2020, 12, 21544-21558. [CrossRef] [PubMed]

11. Omran, D.A.E.H.; Awad, A.H.; Mabrouk, M.; Soliman, A.F.; Aziz, A.O.A. Application of Data Mining Techniques to Explore Predictors of HCC in Egyptian Patients with HCV-related Chronic Liver Disease. Asian Pac. J. Cancer Prev. 2015, 16, $381-385$. [CrossRef] [PubMed]

12. Zhang, R.; Jiang, Y.-Y.; Xiao, K.; Huang, X.-Q.; Wang, J.; Chen, S.-Y. Candidate lncRNA-miRNA-mRNA network in predicting hepatocarcinogenesis with cirrhosis: An integrated bioinformatics analysis. J. Cancer Res. Clin. Oncol. 2019, 146, 87-96. [CrossRef] [PubMed]

13. Shimose, S.; Kawaguchi, T.; Tanaka, M.; Iwamoto, H.; Miyazaki, K.; Moriyama, E.; Suzuki, H.; Niizeki, T.; Shirono, T.; Nakano, M.; et al. Lenvatinib prolongs the progression-free survival time of patients with intermediate-stage hepatocellular carcinoma refractory to transarterial chemoembolization: A multicenter cohort study using data mining analysis. Oncol. Lett. 2020, 20, 2257-2265. [CrossRef] [PubMed]

14. Yang, P.-M.; Lin, L.-S.; Liu, T.-P. Sorafenib Inhibits Ribonucleotide Reductase Regulatory Subunit M2 (RRM2) in Hepatocellular Carcinoma Cells. Biomolecules 2020, 10, 117. [CrossRef] [PubMed]

15. Schwartz, L.H.; Litière, S.; de Vries, E.; Ford, R.; Gwyther, S.; Mandrekar, S.; Shankar, L.; Bogaerts, J.; Chen, A.; Dancey, J.; et al. RECIST 1.1-Update and clarification: From the RECIST committee. Eur. J. Cancer 2016, 62, 132-137. [CrossRef] [PubMed]

16. Joerger, A.C.; Fersht, A.R. Structure-function-rescue: The diverse nature of common p53 cancer mutants. Oncogene 2007, 26, 2226-2242. [CrossRef] [PubMed]

17. Varela, M.; Real, M.I.; Burrel, M.; Forner, A.; Sala, M.; Brunet, M.; Ayuso, C.; Castells, L.; Montañá, X.; Llovet, J.M.; et al. Chemoembolization of hepatocellular carcinoma with drug eluting beads: Efficacy and doxorubicin pharmacokinetics. J. Hepatol. 2007, 46, 474-481. [CrossRef] [PubMed]

18. Fawcett, T. An introduction to ROC analysis. Pattern Recognit. Lett. 2006, 27, 861-874. [CrossRef] 